\title{
Combining Multiscale Filtering and Neural Networks for Local Rainfall Forecast
}

\author{
Fulgencio S. Buendia , Gabriel Buendia Moya, and Diego Andina
}

\begin{abstract}
Rainfall is one of the most important events of human life and society. Some rainfall phenomena like floods or hailstone are a threat to agriculture, business and even life. Predicting the weather has emerged as one of the most important areas of scientific endeavour. Nowadays, there is a big effort and great developments in long and mid-term rainfall forecasts, where qualitative improvements have been obtained both in forecasts and verification. This work proposes a diverse local rainfall forecasting system, using a long term local measurements registry. The forecast is performed estimating pressure time series and processing them with multispectral wavelet analysis and Neural Networks. The aim of the study is to provide complementary criteria based on the observed pressure wave pattern repetition. This method was proposed by expert meteorologists after observing these events during 40 years.
\end{abstract}

Keywords: Rainfall - Forecast - Wavelet - Neural networks · Multispectral analysis

\section{Introduction}

Since climate change is now widely accepted and there is a rapidly increasing realisation that it will affect every person in the world, the resources and efforts in weather investigation have been increased. One of the most important event of daily life is rainfall, predictions became more accurate, as a result weather forecasts has made society more and more dependent on them. Weather predictions are provided by Ensemble Prediction Systems (EPS). A ensemble is a collection of weather forecasts, properly named, are a set of numerical weather prediction (NWP) systems. The ensembles run the models many times from very slightly different initial conditions. Often the model physics is also slightly perturbed, and some ensembles use more than one model within the ensemble (multi-model EPS) or the same model but with different combinations of physical parameterization schemes (multi-physics EPS), see WMO (2012). Some European reference centres are the HIRLAM, ECMWF and the UK MET Office. The European Centre for Medium-Range Weather Forecasts (ECMWF) is supported by 34 states and specialised in prediction for the medium range (up to two weeks 
ahead), on the other side, the HIRLAM (HIgh Resolution Limited Area Model) is a research cooperation of European meteorological institutes that provides a numerical short-range weather forecasting based on an hydrostatic model. The main handicap of medium and long term forecasts, as in any kind of simulation, is that any uncertainty either in the measurements or in the model definition makes the temporal simulation diverge from the real evolution (Lorenz 1963, 2006; Lynch 2006). Since Richardson in 1942, performed the first NWP setting the equations of a particle in the atmosphere, the prediction systems have awesome evolved (Holton 2004; WMO 2012), but have the inherent limitation due to chaotic nature of weather. Regarded to this matter, Rodriguez (2008) stated the need to evolve the current forecast systems to overcome these limitations. Many other approximations based on stochastic models have been proposed (Cowpertwait et al. 1996; Burton et al. 2008). Lovejoy and Mandelbrot proposed a fractal model of rain fields, Lovejoy and Mandelbrot (1985). The use of ANN in weather forecast was first proposed by $\mathrm{Hu}$ (2004), and during last years, several authors have made different approximations to this subject, Dubey performs a good summary of Rainfall prediction using ANN (2015). The work proposes an approximation to local rainfall forecast based weather time series analysis and neural networks to complement current ensemble predictions.

\section{Local Rainfall Forecast Proposal}

\subsection{Local Rainfall Forecast Model}

During many years the observation in the Valladolid weather forecast of rainfall patterns repetition, motivated the registry twice a day of a selected set of data to characterize the rainfall events as an aid to the ensembles evolution prediction. As a result the rainfall events produced in Valladolid as the winds at surface and at $500 \mathrm{Hpa}$ and different pressure waves were recorded in a time series during more of a decade. At a first analysis of the data, it was clearly seen the high dependence of rainfall events and precipitation amount with the combination of surface and height winds. On the other hand, the pressure waves situation can suggest, not so clearly as winds, the rainfall situation. These set of data evolution was used as an aid to the predictions. The idea is to automate the process and, given a situation, being able to predict the evolution of the data, but not by the models, but using the historical registry and a Neural Networks stage. This study started in Buendia et al. (2008). The system retreives the preasure and geostrophic winds from the national meteorological agencies, and filter them to make a forecast of these variables evolution with the historical database. In Buendia et al. (2008) there is explained the first stage of the work, how to filter the input data. Note, the stages of the system are (Fig.1):

- A Capture Stage to retreive the input observations.

- Filtering stage, which prepares the data.

- Historical database.

- A time series forecaster to predict the evolution.

- A classification stage. 


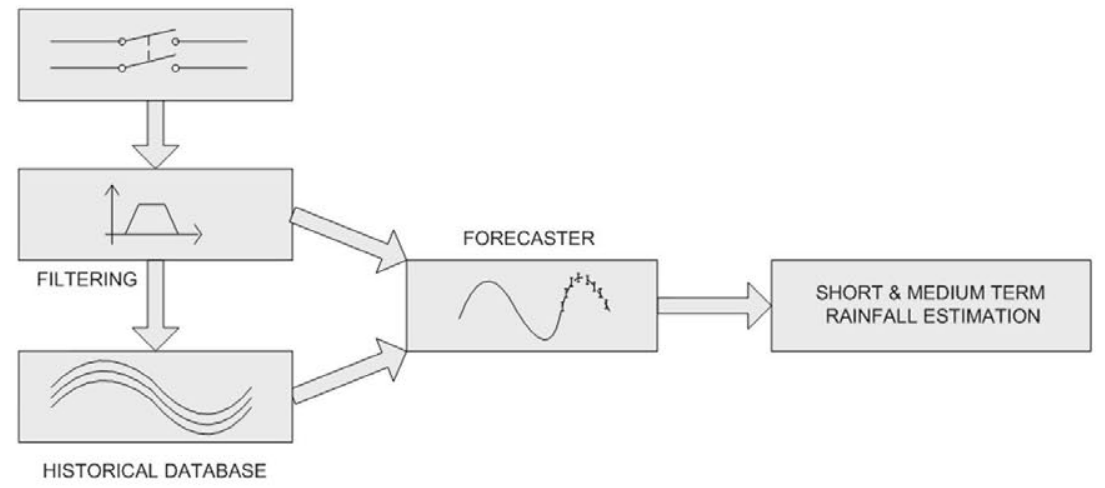

Fig. 1. System architecture

\subsection{Vertical Profile of the Atmosphere Observation Time Series}

As explained before, two sets of variables were selected. The first related to the height between certain pressure levels:

- Pressure waves, measured in hecto pascals, Hpa.

- Geopotential height at $500 \mathrm{Hp}$, measured in metres.

- Thickness of the pressure layer from 500-1000 Hpa, measured in metres.

Plotting these variables among time appear a set of time series, that can be seen as the evolution of the vertical profile of the atmosphere at sea level, 500 Hpa and 1000 Hpa over the observation. The evolution of the data shows the pass through of warm and cold fronts, and the other sinoptic situations, that are directly related to the rainfall events. The data have been captured twice a day during 10 years.

\subsection{Geostrophic Winds Direction Observations}

To complete the dataset, the direction of the geostrophic winds (GW) at sea level and at $500 \mathrm{Hpa}$ has been registered.

The geostrophic wind is the resulting wind in the atmosphere under horizontal and hydrostatic movement without any acceleration or friction, actually at sea level is just theoretical due to the friction forces to the Earth.

As the geostrophic winds are registered each $12 \mathrm{~h}$, this provides a time series that estimates the rainfall probability. This time series will be named $p_{\text {wind }}$. Next figure shows the $p_{\text {wind }}$ probability time series for the eary 1999. It can be cuantitative seen that in the peaks of the signal there are several rainfalls clusters (Fig. 2). 


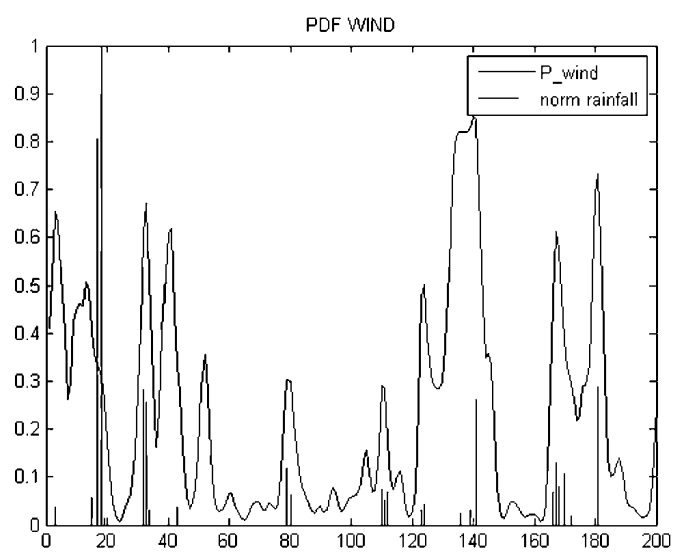

Fig. 2. Rainfall probability time series $p_{\text {wind }}$ with rainfall events

\section{Filtering Stage}

\subsection{Filtering Stage Design}

This stage is designed to simplify and extract the useful information from the input data to:

- Obtain simple data where the rainfall information is contained.

- Obtain simple series easy to forecast individually.

The idea is, the simpler the time series the easier to forecast. So the input signals are decomposed using the multiscale analysis idea, using a family of convolution filters inspired in the time-scale wavelet concept; see Percibal and Walden (2002), Addison (2002), and Mallat (1999). The family of filters, $h(n)$, is a set of band-pass filters obtained subtracting two gaussian distributions:

$$
h(n)=A\left(\exp \left\{-\left(\frac{n 2^{s+2}}{N}\right)^{2}\right\}-\exp \left\{-\left(\frac{n 2^{s+1}}{N}\right)^{2}\right\}\right)
$$

Where $\mathrm{A}$ is a scaling coefficient, $\mathrm{N}$ is the number of input samples, $\mathrm{n}$ is an integer number $(\mathrm{n}=1 . . \mathrm{N})$ and $\mathrm{S}$ represents the "scale" of the filter. This actually is the rest of two gaussian distributions. The responses of this family of filters in the time domain are represented in left side of Fig. 3 and the right figure shows it in the frequency domain. These have a frequency response similar to the Mexican Hat wavelet, Addison (2002).

With the family of filters, the scale used would depend on the temporal scale to observe. The filter, is Shift Invariant (as it is a convolution of a continuous signal), it means that the events represented in the different scales at the same position correspond to the same time instant (which is necessary to locate 

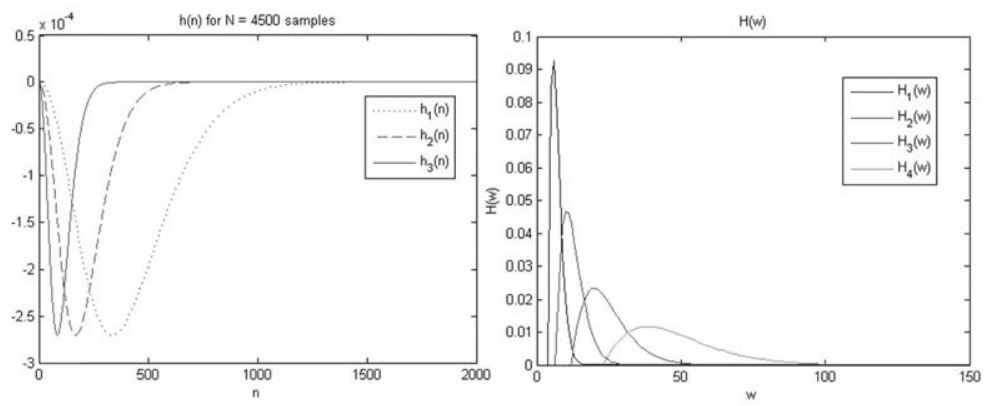

Fig. 3. Time and frequency response of the multiscale filter

events in time series using filtering stages). Some wavelet transforms, as the Continuous Wavelet Transform (CWT), the Maxima Overlap Wavelet Transform (MODWT), the Double Tree Wavelet transform (DWCWT) preserves also the invariance of the event location among the scales, and consequently, can be used. For example, the Discrete Wavelet Transform (DWT) is not shift invariant.

\subsection{Filtering Application: $P_{e s c}, Z_{500}$ and $H_{e s c}$}

Three components can be expected in the time series analysis: an stationery periodic component in the low scales/frequencies, the non-stationary components in the middle and high scales/frequencies range. The stationary components of the pressure are well known due to cyclic contributions, and the high frequency scales the main contribution is due to the day/night pressure variations. The middle range variations can be attributed to frontal circulation.

Figure 4 shows the decomposition of the $P_{\text {esc }}$ for scales 3, 4, 7 and 10 through 2002 to the end of 2011. Those signals are obtained filtering $P_{\text {esc }}$ with the Eq. 1. The filtered signal, at a certain scale, holds the main variations of the signal at a range of time, for instance, scale $2-3$ holds the main variations of the signal in one year scale approximately. The signals at scales 10 and above are quite sharp and therefore discarded.

Figure 5 the resulting of filtering $P_{\text {esc }}$ at scales 7, 8 and 9 . While in scale 7 the variation of the signal is to slow to fit the rainfall clusters, at scales 8 and 9 the signal enclose perfectly. The rainfall is bounded by the valleys of the pressure wave. The decomposition of the other time series $\left(Z_{500}\right.$ and $\left.H_{e s c}\right)$ presents a similar pattern.

In the rest of the article, it is supposed that $P_{e s c}, Z_{500}$ and $H_{e s c}$, are the signals filtered at scale $8+9$. Note that the selection of the scales is referred to the length of the input vector. 

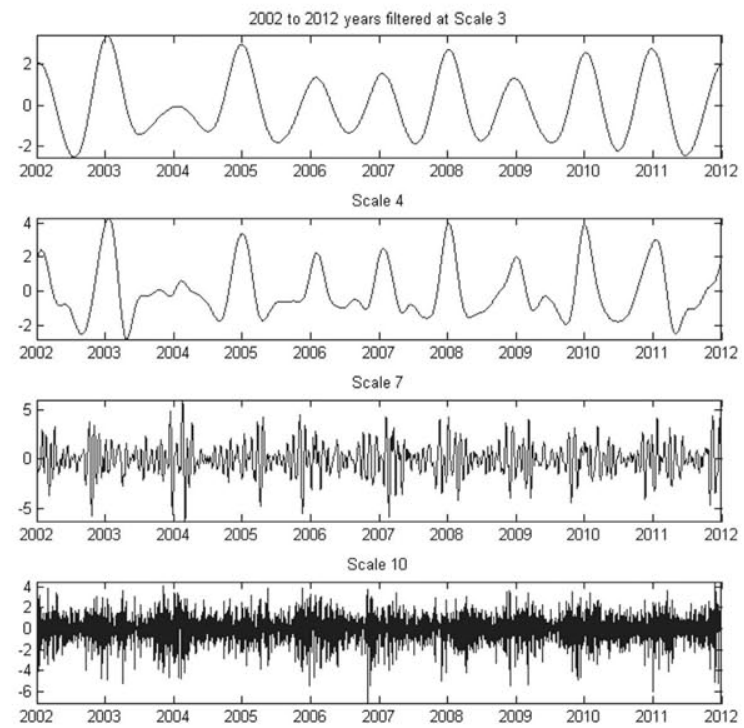

Fig. 4. Multiscale decomposition of $P_{\text {esc }}$ at low and high scales

\section{Classifier Design}

The classification stage tries to discriminate if a certain situation corresponds to a rainfall event or not. The selected classifier was a Multilayer Perceptron (MLP), for its well known classification capabilities.

In the following points there are explained the whole classifier design steps.

\subsection{Labelling}

The first step is create a features vector to use in the classifier. Actually, this is probably the most important step in the classifier design. In this case, the input data are presented as time series from the initial study (scaled pressure waves, geostropic winds and related variables). As the rainfall events are presented in clusters and usually those clusters are enclosed in the pressure valleys, the feature extraction begins obtaining the time series extremes and performs a set of measurements between one maximum and the following one. The data obtained in a valley without rain, shall be labelled as "Dry" and the data found in a pressure valley with rain shall be labelled as "Rain". This method allows to perform an automatic labelling of the data that simplifies the process (see Shasha and Zhu 2004). Situated in each maximum of $P_{e s c}$, there are obtained different parameters. The meaning of these measurements is different for each magnitude but all significative in the rainfall process. All the feature values were normalized to avoid training problems in the following stages. Processed the time series the obtained dataset had 24 features, each of them a training candidate for the classifier. 

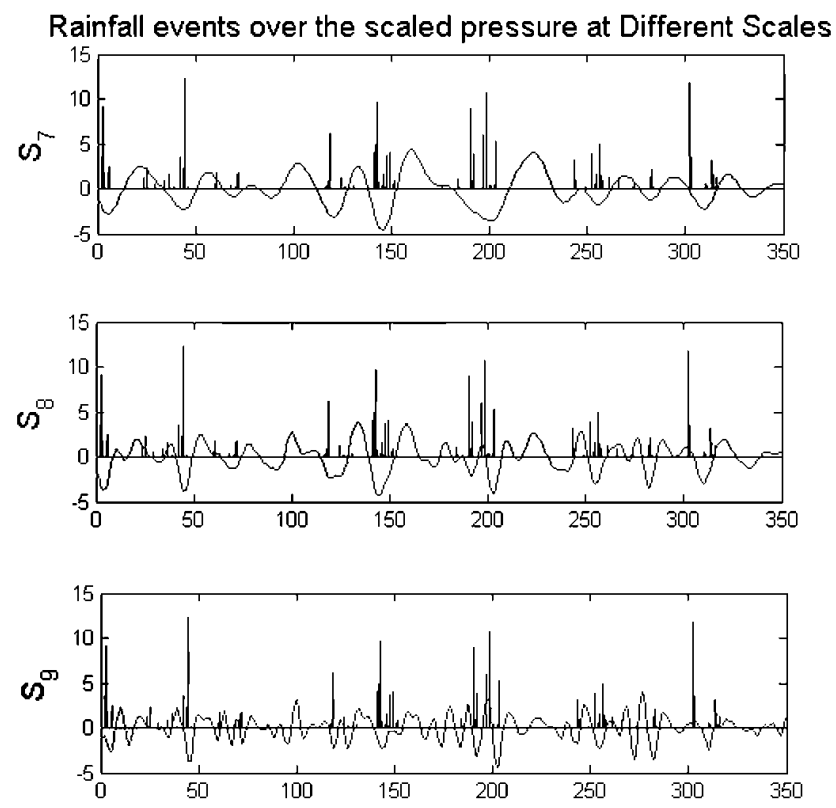

Fig. 5. Multiscale decomposition of $P_{e s c}$ at intermediate scales

\subsection{Feature Extraction}

To perform the feature extraction, a sequential forward selection (SFS) algorithm was applied, as it looks for the best training set. It was used a Multilayer Perceptron as selector, Duda (2000). The SFS process tried to classify 100 samples with the combinations of 24 -features vector. The training results are shown in Fig. 6 . The best training set was: $\left[p_{\text {wind }}, \min P_{E S C}, \Delta Z_{500}, \Delta H_{e s c}\right]$. The error rises when additional features added, so the process was stopped there.

As a trade off between performance and complexity, the first two features were included in the training vector, since these features need to be forecasted. The other two variables will be included later when the system start to work.

\subsection{Test Vectors Preparation}

With the selected features, the data needs to be introduced in the Multi Layer Perceptron. The matrix with the input variables is usually called $P_{\text {matrix }}$, and the target vector $T_{\text {vector }} . P_{\text {matrix }}$ holds $\left[p_{W I N D}, \min P_{E S C}\right]$ sample and $T_{\text {vector }}$ 1 or 0 , indicating if the $P_{\text {data }}$ corresponds to "Rain" and 0 to "Dry". The dataset is divided into three subsets: Training dataset, for perform the training of the MLP - (185 vectors). Verification dataset: (185 vectors). Test dataset: Data to test the final classification (300 vectors) (Fig.6).

The first two datasets are used to setup and train the classifier and the last one is used to test the classifier performance. 


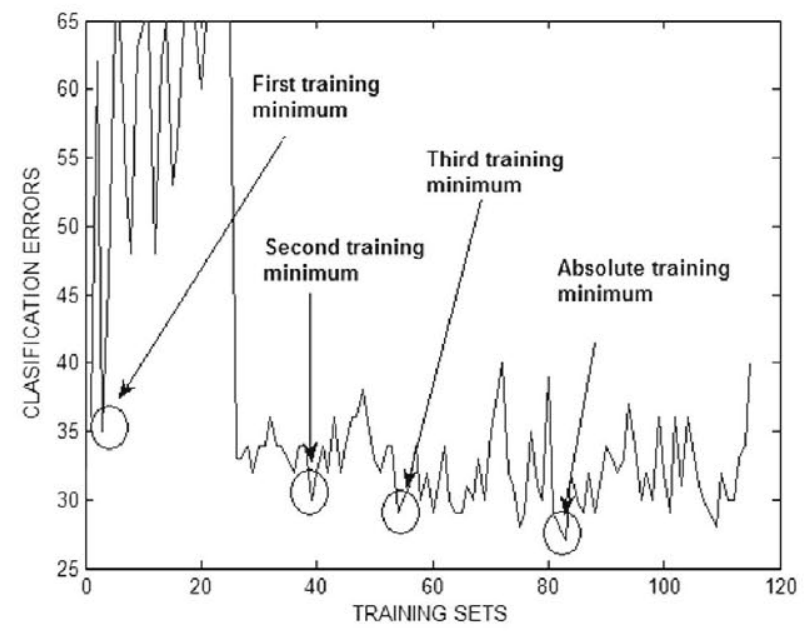

Fig. 6. SFS process

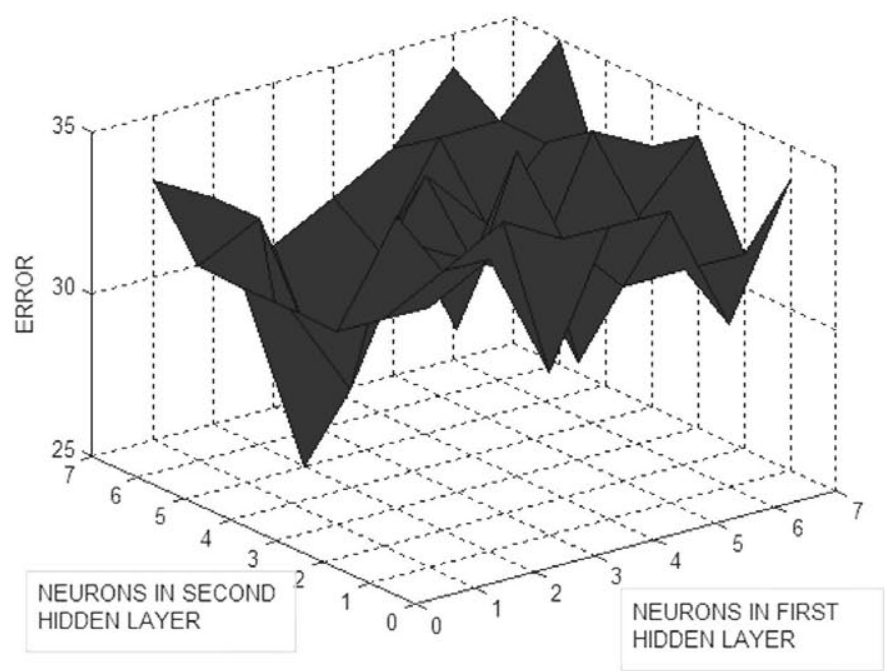

Fig. 7. MLP hidden layers selection

\subsection{Classifier Setup and Training}

It was selected a multilayer perceptron (MLP) with two hidden layers, as can generate arbitrarily complex boundaries to classify vectors into classes consisting of any union of polyhedral regions (Bishop 1996). There is no rule to select the optimal number of neurons for a MLP, some guidelines can be found in Duda (2000), etc. It was tested every configuration of a two-hidden layer network, from $1 \times 1$ neurons to $7 \times 7$ neurons, selecting the one with minimum mean squared error (MSE) (Fig. 7). 
The best performance was achieved with two neurons in the first hidden layer and five in the second, with a classification performance of $87 \%$ (25 classification failures in the 185 verification samples). The training was repeated with the network until the number of errors was below $10 \%$ of MSE, at the end the network achieved 19 failures in the 185 verification samples, that means a $90 \%$ of a priori performance. This value needed to be finally confirmed with the test dataset. 4.5 Classifier performance assessment The network was tested with the test dataset, with vectors not previously used neither to train nor verify the classifier. It appeared 36 failures in 300 samples, that is a $88 \%$ of performance. In Table 1 all these data are summarized.

Table 1. Classification performance summary

\begin{tabular}{l|l|l}
\hline & Verification dataset & Test dataset \\
\hline Number of samples & 185 & 300 \\
\hline Number of failures & 19 & 36 \\
\hline Classification performance & $90 \%$ & $88 \%$ \\
\hline
\end{tabular}

\section{Conclusions}

This paper presents a complementary method to current forecasts provided by ensembles, as the rainfall is predicted using artificial neural networks and time series. It has been presented the study of the meteorological variables for the local rainfall study, explaining the filtering and classification stages. At the moment, it has been obtained $88 \%$ of classification performance that is quite successful. Currently, it is being developed the time series forecast, with promissory results. The accuracy and prediction range shall determine the overall system performance. The work continues the presented one in Buendia et al. (2008).

\section{References}

Addison, P.S.: The Illustrated Wavelet Transform Handbook, Introductory Theory and Applications in Science, Engineering, Medicine and Finance, 1st edn., 368 p. Taylor \& Francis, Abingdon (2002)

Bishop, C.: Neural Networks for Pattern Recognition, 1st edn., 504 p. Oxford University Press, Oxford (1996)

Buendia, F.-S., Tarquis, A.M., Buenda, G., Andina, D.: Feature extraction via multiresolution MODWT analysis in a rainfall forecast system. In: World Multiconference on Systemics, Cybernetics and Informatics 2008 Proceedings, pp. 69-73 (2008)

Burton, A., Kilsby, C.G., Fowler, H.J., Cowpertwait, P.S.P., OConnell, P.E.: RainSim: a spatial temporal stochastic rainfall modelling system. Environ. Model Softw. 23, 1356-1369 (2008) 
Cowpertwait, P.S.P., OConnell, P.E., Metcalfe, A.V., Mawdsley, J.A.: Stochastic point process modelling of rainfall, I. Single site fitting and validation. J. Hydrol. 175, $17-46$ (1996)

Dubey, A.D.: Artificial neural network models for rainfall prediction in Pondicherry. Int. J. Comput. Appl. (0975 - 8887) 120(3), 30-35 (2015)

Duda, R.O., Hart, P.E., Stork, D.G.: Pattern Classification, 2nd edn., 680 p. Wiley, New York (2000)

Holton, J.R.: An Introduction To Dynamic Meteorology, 4th edn., 534 p. Academic Press, Cambridge (2004)

Hu, M.J.-C.: Application of the adaline system to weather forecasting. Dissertation Department of Electrical Engineering, Stanford University (1964)

Lorenz, E.N.: The predictability of hydrodynamic flow. Trans. N. Y. Acad. Sci. Ser. II 25(4), 409-432 (1963)

Lorenz, E.N.: Predictability, a problem partially solved. In: Palmer, T., Hagedorn, R. (eds.) Predictability of Weather and Climate, pp. 40-58. Cambridge University Press, Cambridge (2006)

Lovejoy, S., Mandelbrot, B.: Fractal properties of rain, and a fractal model. Tellus A 37 A, 209-232 (1985)

Lynch, P.: Chaos, predictability and ensemble forecasting. In: The Emergence of Numerical Weather Prediction: Richardson's Dream, pp. 229-234. Cambridge University Press (2006)

Mallat, S.: A Wavelet Tour of Signal Processing, 2nd edn., 620 p. Academic Press, Elsevier, Cambridge (1999)

Percibal, D.B., Walden, A.: Wavelet Methods for Time Series Analysis, 6220 p. Cambridge University Press, Cambridge (2002)

Rodriguez, M.A.: Predicción Meteorológica y Caos en Espacio-Tiempo (Spanish). Revista Española de Física 22, 66-69 (2008)

Shasha, D., Zhu, Y.: High Performance Discovery in Time Series, Techniques and Case Studies. Monographs in Computer Science. Springer, Heidelberg (2004)

World Meteorological Organization: Guidelines on Ensemble Prediction Systems and Forecasting. WMO No-1091 (2012) 